\title{
Commensally sourced antibiotics
}

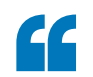

lactocillin

was active against several pathogens
The potential of the microbiome to influence human health and disease is now well-recognized, but the diversity of microbial products that are produced by a healthy microbiota is largely unexplored. Donia et al. have carried out the first systematic screen of biosynthetic gene clusters (BGCs) in the healthy human microbiota and found that BGCs for the thiopeptide class of antibiotics are widely distributed. Interestingly, they also revealed that the novel thiopeptide lactocillin is produced by a vaginal commensal bacterium and has antibacterial activity.

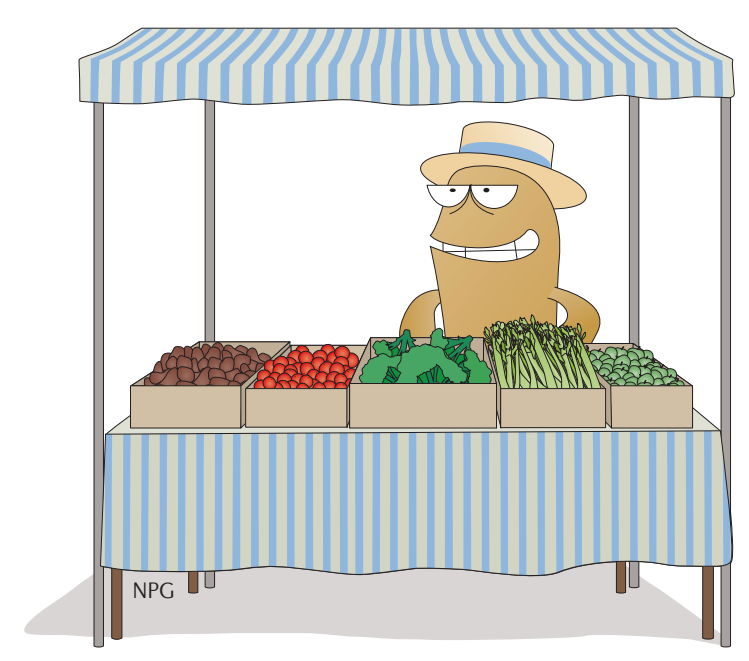

To identify novel microbiotaderived products, Donia et al. analysed the reference genomes of 2,430 human-associated bacteria for the presence of BGCs involved in the synthesis of a range of small molecules. By examining metagenomic samples from the Human Microbiome Project, they found that 3,118 of the 14,000 identified candidate BGCs were expressed in the microbiota of healthy individuals. The abundance of BGCs varied with anatomical location; more BGCs were present in the gut and the oral cavity than in the skin, airways and urogenital tract. Notably, although some BGCs were restricted in their distribution, BGCs that encode the thiopeptide class of antibiotics were present at all anatomical sites.

To investigate the potential functional role of one of the abundant thiopeptide BGCs, the authors purified and characterized the product of the bgc66 cluster encoded by Lactobacillus gasseri, a predominant member of the healthy vaginal microbiota. Using a combination of nuclear magnetic resonance, mass spectrometry and isotype labelling experiments, the authors found that the product of bgc66 was similar to the Bacillus cereus thiopeptide thiocillin, and it was named lactocillin. The antibacterial capacity of lactocillin was then tested in vitro against several common pathogens and commensals from the vaginal community. Interestingly, lactocillin was active against several pathogens (including Staphylococcus aureus, Corynebacterium aurimucosum and Gardnerella vaginalis) but was inactive against commensals (including Lactobacillus spp. and Streptococcus spp.). Finally, metatranscriptomic data analysis revealed that thiopeptide BGCs are expressed in vivo in human samples.

Collectively, these data establish a new strategy for the identification of novel microbiota-derived molecules and, although the role of lactocillin in vivo needs further investigation, these findings highlight the widespread distribution of microbial small molecules that have the potential to mediate interactions between members of the microbial community and with the host.

Cláudio Nunes-Alves, Associate Editor, Nature Reviews Microbiology This article is modified from the original in Nature Rev. Micro (http://dx.doi.org/10.1038/ nrmicro3363)

ORIGINAL RESEARCH PAPER Donia, M. S. et al. A systematic analysis of biosynthetic gene clusters in the human microbiome reveals a common family of antibiotics. Cell 158, 1402-1414 (2014) 